\title{
Herbal Photoprotective Formulations and their Evaluation
}

\author{
Deep Chanchal and Saraf Swarnlata*
}

Institute of Pharmacy, Pt. Ravishankar Shukla University, Raipur., C.G. 492001, India

\begin{abstract}
Photochemoprevention has become an important armamentarium in the fight against ultraviolet radiation induced damage to the skin. UV irradiation to skin results in erythema, edema, sunburn cells, hyperplasia, immunosuppression, photoaging and photocarcinogenesis. Various synthetic agents have been used as photoprotectives but they have limited use because of their potential toxicity in humans and their ability to interfere only in selected pathways of multistage process of carcinogenesis. Several botanical compounds have been shown to be antimutagenic, anticarcinogenic and nontoxic and have ability to exert striking inhibitory effects on a plethora of cellular events at various stages of carcinogenesis. Since multiple pathways are involved in photocarcinogenesis so mixture of several botanical antioxidants working through various mechanisms, in conjunction with the use of sunscreens could also be an effective approach for reducing photoaging and skin cancer in humans. The performance of sunscreen substances could be improved by modification of their chemical, physical and technological properties or by the use of novel carriers like liposomes, nanoparticles, phytosomes, transferosomes, nanospheres etc. The application of novel approaches can also improve its efficacy regarding continuous action of herbs on the human body. This review will focus on list of herbs, formulations and evaluation parameters of photoprotective herbs.
\end{abstract}

Keywords: Photoprotectives, evaluation, herbs, photoaging, herbal formulations.

\section{INTRODUCTION}

Long exposure of UV radiation increases the risk of skin cancer including basal cell and squamous cell carcinoma as well as malignant melanoma. There are also many specific diseases like phototoxic or photoallergic reactions, autoimmune diseases including lupus erythematous, idiopathic photodermatosis and varieties of skin cancers which are triggered or exacerbated by UV radiation exposure. Solar UVR is divided into three categories UV-C (200-280 nm), UV-B $(280-320 \mathrm{~nm})$ and UV-A (320-400 nm ). UV-C is most biologically damaging but it is filtered out by ozone layer. Currently UV-B radiation and to a lesser extent UV-A are responsible for inducing skin cancer [1]. Among many UV-R damages, skin cancer is of greatest concern as its rates have been increasing. Ultraviolet radiation increases oxidative stress in skin cells by causing excessive generation of reactive oxygen species (ROS), leading to cancer initiation and promotion. The main histological features of photoaging include dermal damage with marked elastotic degenerative change, loss of collagen, reduction in number and size of fibroblasts, an increase in proteoglycans and a moderate mononuclear inflammatory cell infiltrate. Irregular epidermal thickening progressing in the later stages leads to dysplasia and cytological atypia [2]. Development of novel strategies to reduce the occurrence of skin cancer and delay the process of photoaging are highly desirable goals. Photoprotection could be achieved by the use of sunscreens, moisturizers, keratolytics and antioxidants.

There are so many synthetic sunscreen agents (Octabenzone, Octyl methoxyciinnan ate, Benzophenone-3, Pro-

*Address correspondence to this author at the Institute of Pharmacy, Pt. Ravishankar Shukla University, Raipur., C.G. 492001, India; Tel/Fax: +91 771 2262832; E-mail: swarnlata_saraf@rediffmail.com vatene, 2-Ethoxy Ethyl P-Methoxycinnamate, Sulisobenzone, Mexenone, Avobenzone, Dioxybenzone, 4-Dimethyl Amino Benzoic acid etc.) are available in form of photoprotectives with certain limitation, which restrict their use at cellular level. They have limited use because of their potential toxicity in humans and their ability to interfere only in selected pathways of the multistage process of carcinogenesis [3]. Botanical agents may work in various ways by stimulating the immune response, by inducing gene suppression, by detoxifying carcinogens, by blocking oxidative damage to DNA, by initiating selected signaling pathways or by other mechanisms. Thus many of these agents play multiple roles in ameliorating the process of carcinogenesis [3]. (Fig. 1) Few examples include tea polyphenols, curcumin, silymarin, garlic compounds, apigenin, resveratrol, ginkgo biloba, beta- carotenoids, ascorbic acid etc. Compounds that can protect against UV- A and UV- B radiations both could be ideal photochemoprotective agents [3].

The active synthetic molecules are being used since years, but the research shows that these active molecules adversely affects on human skin via self inducing reactive oxygen species. Thus, to overcome such serious side effects of synthetic molecules, the research is now diverted towards natural biomaterials [4]. Naturally occurring antioxidants like alpha carotene, ascorbic acids, flavones, flavanone, have ability to donate electrons and stops free radical chain reactions and also showed broad spectrum UV absorption $[5,6]$ It was reported that association of botanical extracts (bioflavonoids) and vitamins produce synergistic photoprotective effects in preventing increased erythema, transepidermal water loss and sunburn cell formation [7].

The herbs as biological additives in form of extracts are utilizing since long period of time in the cosmetic formulation and now scientific evidences proven that many plant 

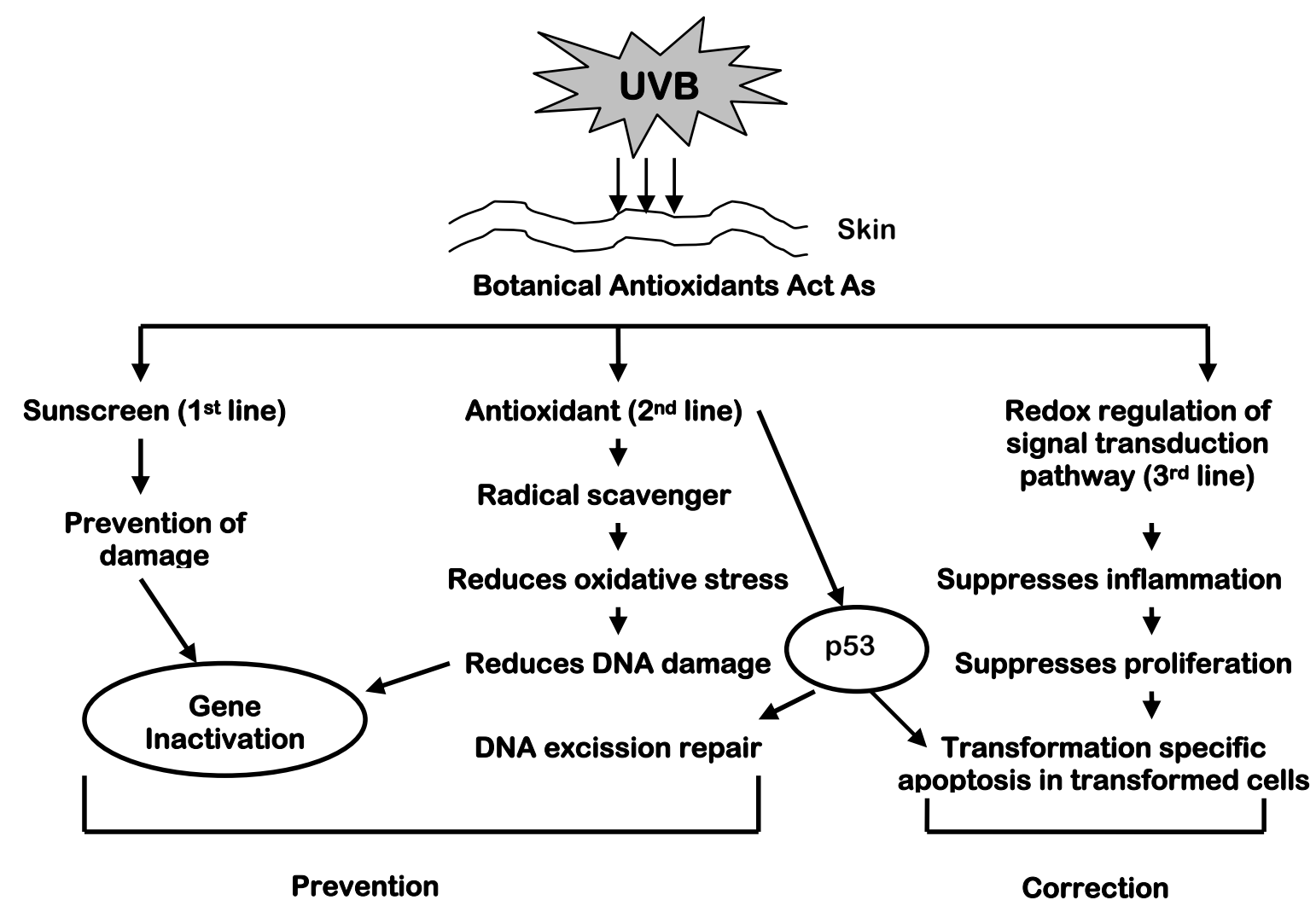

Fig. (1). Multiple pathway mechanism of botanical photoprotectives [3].

extracts showed their photo protective activity with significant improvement in enzymes like superoxidedismutase, catalase and total protein and ascorbic acid level [8]. Extracts of many plants, citrus fruits and leafy vegetables as source of ascorbic acid, vitamin $\mathrm{E}$ and phenolics compounds and enzymes possess the ability to reduce the oxidative damage. These oxidative damages ultimately reduce the protective enzyme level and imbalances the level of total protein and ascorbic acid and other antioxidants level in cells. The creams comprises such extracts could be utilized for the protection of photo induced intrinsic oxidative stress as well as structural alteration in skin [9].

In the series of natural photo protective agents, enzymes like superoxide dismutase (SOD), peroxidase and proteolytic enzymes have opened up new avenues to photoprotective reaction occurring within the cell. These chemical entities were first discovered in 19th century, where as use of enzymes in cosmetics have been advocated for many years [10]. Proteolytic enzymes like bromelaine, papain etc. has been used for skin peeling and smoothing [11]. These are utilized as efficient tool to reduce UV-induced erythema, which can also be thought of as free radical scavenging ability [12].

Naturally occurring herbal compounds such as phenolic acids, flavonoids and high molecular weight polyphenols are very useful for prevention of the adverse effects of UV radiation on the skin and evaluation of their clinical efficacy is awaited [13]. Adequate UV A protection and an appropriate testing method of UV A efficacy are the most pressing unmet needs [14]. Most commonly used herbal photoprotectives are listed in Table $\mathbf{1}$, this table will be helpful in the selection of herbal constituent for formulation of photoprotective dosage form.

\section{HISTOPATHOLOGICAL CHANGES OF PHO- TOAGING}

In skin aging two independent processes occur simultaneously (i) Chronological or intrinsic aging which affects the skin by slow and irreversible tissue degeneration and (ii) extrinsic aging or photoaging, which results from exposure to environmental factors including primarily ultraviolet (UV) radiation. In chronological aging skin becomes thin, dry, pale and exhibits fine wrinkles while, photoaging leads to marked cutaneous alterations characterized clinically by deep wrinkles, roughness, sallowness, mottled dyspigmentation, telangiectasia and a variety of benign and malignant neoplasms. In chronologically aged skin, the different characteristic steps of epidermal differentiation are preserved, however, based on a reduced keratinocyte proliferation, epidermal thinning is observed histologically. The dermis is also thin in aged skin, resulting from a reduction in the amount and organization of connective tissue. The extracellular matrix in the dermis is composed primarily of type I collagen, associated with type III collagen, elastin, proteoglycans and fibronectin. Sun-exposed sites usually display a reduction in mature type I collagen and an increase in collagen III-tocollagen I ratio. The histopathological hall-mark of photoaging is a massive accumulation of elastotic material in the upper and mid dermis also known as solar elastosis. Elastosis is due to accumulation of damaged elastin, the main component of elastic fibers, and is associated with degeneration of the surrounding collagen meshwork [15]. The histological effects can be well studied by Table 2, it helps to decide the evaluation parameter (Table 3). 
Table 1. Herbal Constituents Used as Photoprotectives [2, 13]

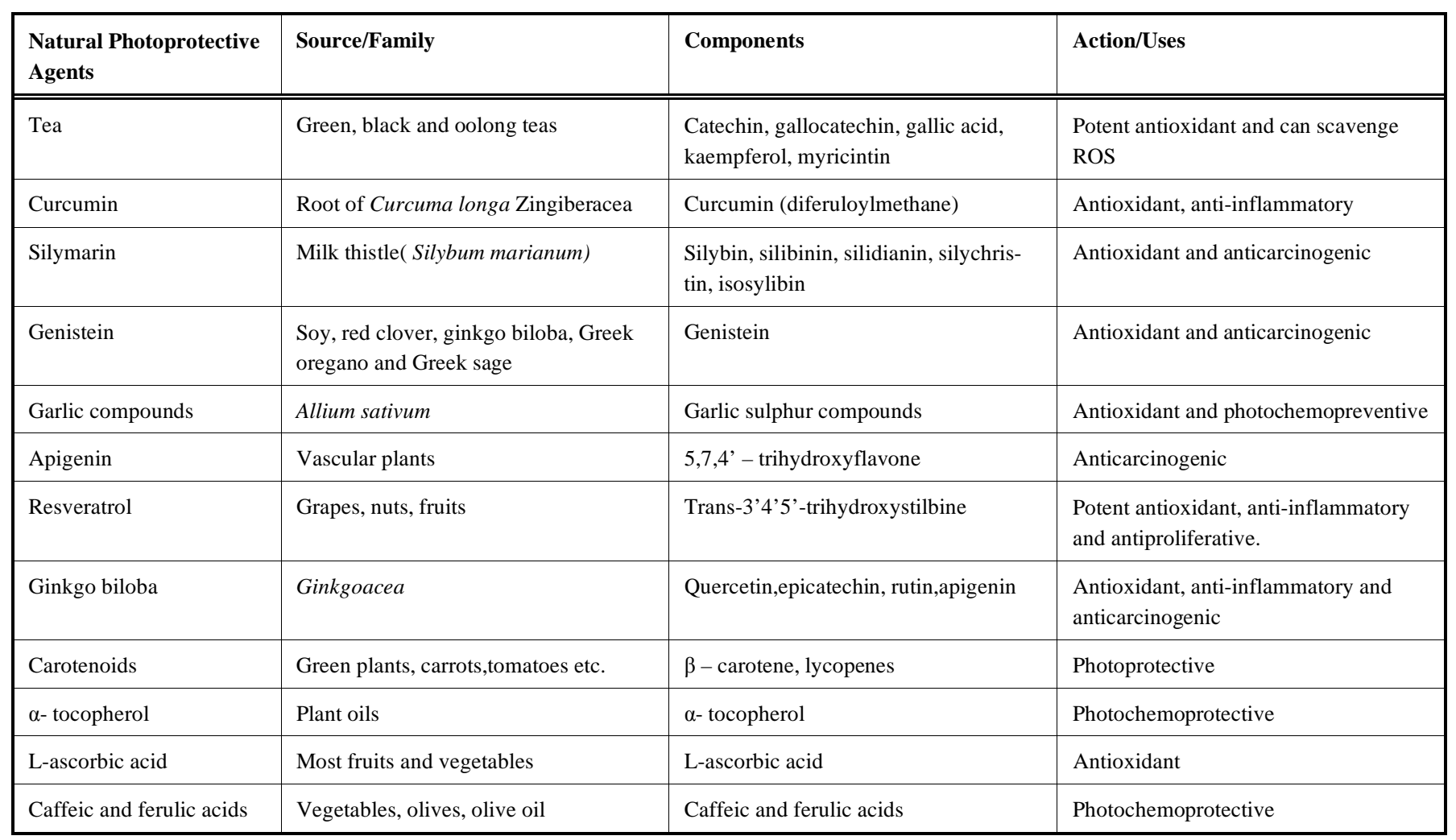

Table 2. Histopathological Effects of Photoaging [16]

\begin{tabular}{|l|l|}
\hline Parameters & Changes in Photoaged Skin \\
\hline \hline Number of keratinocytes & Reduces \\
\hline Number of fibroblasts & Reduces \\
\hline Epidermal cells & Get flattened \\
\hline Number of Langerhans cells and melanocytes & Decreases \\
\hline Lymphatic channels of dermis & Dilates \\
\hline Collagen and Elastic fibers & Reduces \\
\hline Fat content & Loses and redistributes \\
\hline Pigmented cells & Overactive causing blotches of hyperpigmentation \\
\hline
\end{tabular}

\section{PHOTOPROTECTIVE FORMULATIONS}

Commonly used cosmetics have low affinity to the skin and have little percutaneous absorption. The conventional cosmetics show little efficiency as a cosmetic. Novel technology has shown great potential for improving the effectiveness and efficiency of delivery of nutraceuticals and bioactive compounds. Recent advances in nanotechnology show their promise as potential cosmetics for poorly soluble, poorly absorbed and labile herbal extracts and phytochemicals. An innovative approach can improve both the aesthetics and performance of a cosmetic product. The application of novel approaches can also improve its efficacy regarding continuous action of herbs on the human body [17]. The formulation and selection of approach to be used for herbal cosmetics will depend upon purpose of preparation that is for topical or systemic effect, inherent properties of drug or herb extract such as hydrophilic or hydrophobic; surface characteristics of a system like permeability and charges; degree of biodegradability, biocompatibility, toxicity; release profile, size of the product required and antigenicity of the final product [18].

The novel approaches that could be used include Microemulsions [19-21], multiple emulsions [22-24], liposomes $[25,26]$, phytosomes [27-32], transfersomes [33-39] noe- 
mulsions [40] multiple nanoemulsions [41], nanocrystals [42], cubosomes [43] and transdermal Delivery System [44].

\section{EVALUATION PARAMETERS OF PHOTOPROTEC- TIVES}

\section{Erythema Determination}

Erythema can be determined by investigating the histological , ultrastructural, biochemical and immunological effects of UV radiation on skin and its relationship to photodamage and skin cancer.

\section{Method}

Hairless mouse model could be used for photo protection studies. Prior to experimentation four hairless mice are anesthetized (using ketamine) and a rectangular area approximately $2.5 \mathrm{~cm} \mathrm{x} 4 \mathrm{~cm}$ is marked off on the dorsal area of each animal. Then sunscreen formulations are applied and one mice is left as control mice which is untreated. After 15 min drying period, ultraviolet radiation are produced by a planar array of two UV- A 340 fluorescent lamps which stimulate UV radiation present in sunlight from wavelength $295 \mathrm{~nm}$ to $365 \mathrm{~nm}$. Irradiance could be measured using Erythema UV A and UV B Intensity meter and minimal erythemal dose (MED) is calculated. One MED is defined as the amount of UV radiation necessary to cause a slight reddening of the skin $24 \mathrm{hr}$ after exposure. For hairless mouse one MED is approximately $140 \mathrm{~m} \mathrm{~J} / \mathrm{cm}^{2}$ [45].

\section{Lipid Damage Determination}

UV radiation induces the formation of reactive oxygen species resulting in damage to various components of skin like lipid damage which is oxidative degradation of unsaturated free fatty acids and cholesterol. It is observed that UV exposure decreases lipid melting temperature of the mouse skin and that application of sunscreens prior to UV radiation would reduce this epidermal damage.

\section{Method}

Immediately following UV exposure, mice are sacrificed by carbondioxide asphyxiation and full thickness, dorsal skin is removed by blunt dissection. The epidermis is separated from the full thickness skin by placing the skin dermis side down on filter paper saturated with $2.5 \%$ (w/v) trypsin solution. After storage at $37{ }^{\circ} \mathrm{C}$ for $4 \mathrm{hr}$, epidermal sheets are gently lifted from the skin using forceps and then covered with fresh trypsin solution and stored at $37{ }^{\circ} \mathrm{C}$ for $1 \mathrm{hr}$. Remove trypsin from epidermal sheets with gentle rinsing using deionized water. Epidermal samples are stored at room temperature and $75 \%$ relative humidity overnight. Then $10 \mathrm{mg}$ samples of epidermis are sealed in aluminium pans and analyzed using Differential scanning colorimeter. Scan the samples at a heating rate of $5{ }^{\circ} \mathrm{C}$ per min from $10{ }^{\circ} \mathrm{C}$ to $80{ }^{\circ} \mathrm{C}$ with a temperature modulation of $0.759{ }^{\circ} \mathrm{C}$ per min and determine the lipid melting temperatures [45].

\section{Edema Determination}

Inflammation is acute biological response to UV radiation. The vasodilatation of cutaneous blood vessels results in erythema (reddening) and edema (swelling). The treatment reduces this edema thus it is also an evaluation parameter for photoprotectives.

\section{Method}

Immediately prior to irradiation, the thickness of skin folds, at the back of the neck of two sedated mice are measured using a spring loaded pocket thickness gauge. Three measurements are taken for each mouse and these data are used as the baseline. Twenty four hours post- UV exposure, mice are sacrificed by carbondioxide asphyxiation and skin fold thickness is measured. Edema is calculated by the difference in skin fold thickness between the baseline and postUV exposure data [45].

\section{Sunburn Cell Count}

\section{Objective}

Sunburn cells are apoprotic keratinocytes that have absorbed a lethal dose of UV radiation and have been observed in humans, mice, rabbits and guinea pigs. They are indication of acute photodamage.

\section{Method}

After measurement of postexposure edema, UV exposed dorsal skin of each mouse is removed and fixed in $10 \%$ formalin solution. two nonsequential sections of the skin are removed and mounted to a slide and Hematoxylin and Eosin stained. The stained samples are then subjected to microscopic examination (600X) and the number of sunburn cells per linear centimeter are calculated. Counts are done on 1.5 $\mathrm{cm}$ sections of interfollicular epidermis [45].

\section{SPF Determination}

SPF by definition is determined in vivo as the increase in exposure time required to induce erythema. Ratio of UV doses protected to unprotected gives the SPF. The in vitro method measures the reduction of the irradiation by measuring the transmittance after passing through a film of product [46].

\section{Method}

The most common invitro technique involves measuring the spectral transmittance at UV wavelengths from $280 \mathrm{~nm}$ to $400 \mathrm{~nm}$. The invitro SPF is calculated as follows:

$$
\begin{aligned}
& \mathrm{SPF}=\int_{200 \mathrm{~nm}}^{400 \mathrm{~nm}} E_{\lambda .} S_{\lambda} \cdot d_{\lambda} \\
& \qquad 00 \mathrm{~nm} \\
& \int_{\lambda} \mathrm{E}_{\lambda} \cdot \mathrm{S}_{\lambda} \cdot \mathrm{T}_{\lambda} \mathrm{d}_{\lambda} \\
& 200 \mathrm{~nm}
\end{aligned}
$$

where,

$\mathrm{E}=\mathrm{CIE}$ erythemal spectral effectiveness

$\mathrm{S}=$ solar spectral iradiance

$\mathrm{T}=$ spectral transmittance of the sample

\section{Quantification of UV Induced DNA Damage}

To estimate whether the application of the test formulation exhibits a protective effect on DNA lesions. 
Table 3. Bioengineering Methods of Evaluation [47]

\begin{tabular}{|l|l|l|}
\hline S.No. & Parameter of Evaluation & Instrument Used \\
\hline \hline 1 & Skin hydration & Corneometer \\
\hline 2 & Skin color & Chromameter \\
\hline 3 & Skin Elasticity & Cutometer \\
\hline 4 & Barrier Function Assessment & Evaporimeter \\
\hline 5 & Skin surface lipid measurement & Sebumeter \\
\hline 6 & Skin roughness and wrinkle & Skin visiometer \\
\hline 7 & Transepidermal water loss (TEWL) & Tewameter \\
\hline 8 & Erythema index & Reflectance Spectrophotometry using Mexameter \\
\hline
\end{tabular}

\section{Method}

The test formulations are applied to volunteers twice daily. After a two week treatment three test areas are established on the inner forearms of every volunteer and marked with a stencil. Five and $24 \mathrm{hr}$ prior to isolation of suction blister epidermis, two areas on every forearm are irradiated with 1.2 minimal erythemal dose solar simulated radiation. The third area is not irradiated and used as a control. One forearm is treated as described above; the colateral forearm is left untreated. To assess DNA damage by single-cell gel electrophoresis (Comet assay), epidermal keratinocytes are isolated from suction blister epidermis and embedded in low gelling agarose gels [47].

\section{Skin Viscoelasticity Determination}

To determine effects of a treatment with the test formulation on skin firmness.

\section{Method}

Two test areas are marked on the forearms of the volunteers. One test area is treated twice daily with the test formulation; the second is left untreated and used as a control. At the baseline visit and after 1,2, and 4 weeks of regular use, skin viscoelasticity is determined using a cutometer. As an additional means of control for volunteer compliance, skin moisture is examined using a corneometer [47, 48].

\section{Wrinkle Volume Determination}

To determine a decrease in wrinkle volume after treatment with the test formulation.

\section{Method}

Volunteers treated the assigned side of the face for 4 weeks twice daily with the test formulation. At the baseline visit and after 4 weeks of treatment, facial wrinkles in the crow's feet area are evaluated by means of in vivo topometry using phase shift rapid in vivo measuring of human skin phase induction PRIMOS. The PRIMOS system represents an established and widely used method to quantify effects on skin wrinkles [47].

\section{Epidermal Cell Turnover Determination}

The decrease in corneocyte size is correlated with accelerated epidermal turnover.

\section{Method}

Three test areas are established on the inner forearms of each volunteer, and a stencil is used to mark these test areas. Two areas are treated with the test formulations, and one area is left untreated and served as additional control. Volunteers apply the test formulations twice daily (morning and evening) for 6 weeks. The size of single corneocytes is determined at the baseline visit and after 4, 5, and 6 weeks of treatment. Ten to $20 \mathrm{~h}$ after application of test samples, measurements are performed. The determination is carried out by image analysis of D-Squame ${ }^{\circledR}$ sheets [47].

\section{CONCLUSION}

The photoprotective formulation must be such that it is stable pharmaceutically and is able to reverse some of the characteristic signs of the chronological aging and photoaging in both skin compartments after topical applications. Various chemical photoprotectives are available but researches are going for use of more herbal constituents as photoprotectives and there is need of better evaluation parameters for those formulations. We have studied above evaluation parameters but more researches have to be carried out to validate simple, economic and rapid methods to carry out efficacy studies of herbal photoprotective formulations. According to the present scenario there is need for adoption of new alternative methods to ensure the safety of cosmetic preparations without or minimum use of animals.

\section{ACKNOWLEDGEMENTS}

One of the author wishes to thank AICTE (RPS scheme) and UGC (Major project), New Delhi for financial support for this work.

\section{REFERENCES}

[1] Afaq, F.; Mukhtar, H. Botanical antioxidants in the prevention of photocarcinogenesis and photoaging. Exp. Dermatol., 2006, 15, 678-684.

[2] Griffiths, C.E.M.; Maddin, S.; Weidow, O.; Marks, R.; Donald, A.E.; Kahlon, G. Treatment of photoaged skin with a cream containing $0.05 \%$ isotretinoin and sunscreens. J. Dermatol. Treat., 2005, 16, 79-86.

[3] Guyer, S.F.; Afaq, F.; Mukhtar, H. Photochemoprotection of skin cancer by botanical agents. Photodermatol. Photoimmunol. Photomed., 2003, 19, 56-72.

[4] Ashawat, M.S.; Saraf, S.; Saraf, S. Antisolar activity of $R$. damnesia and T. errecta. Planta Indica, 2005, 2, 26-28 . 
[5] Bajpai, M.; Parade, A.; Tiwari, S.K.; Prashad, D. Phenolic content and antioxidant activity of some food and medicinal plants. Int. J. Food Sci. Nut., 2005, 4, 287-291.

[6] Ashawat, M.S.; Saraf, S.; Saraf, S. Cosmetic potentiality of plant extracts and natural oils. Biosci. Biotechnol. Res. Asia, 2006, 3, 181-188.

[7] Campos, P.M.B.G.M.; Gianeti, M.D.; Kanashiro, A.; Lucisano, V.Y.M.; Gaspar, L.R. In vitro antioxidant and in vivo photoprotective effects of an association of bioflavonoids with liposoluble vitamins. Photochem. Photobiol., 2006, 82, 683-688.

[8] Ashawat, M.S.; Saraf, S.; Saraf, S. Biochemical and histopathological studies of herbal cream against UV radiation induced damage. Trends Med. Res., 2007a, 2(3), 135-141.

[9] Ashawat, M.S.; Saraf, S.; Saraf, S. In vitro antioxidant activity of ethanolic extracts of Centella asiatica, Punica granatum, Glycyrrhiza glabra and Areca catechu. Res. J. Med. Plants, 2007b, 1, 1316.

[10] Cleaver, J. Defective repair replication of DNA in xeroderma pigmentosum. Nature, 1968, 372, 773-775.

[11] Yarosh, D.J.; Klein, A.; Connor, J.; Hawk, E.; Rafel, P.W. The xeroderma pegmentosum study group: Effect of topically applied $\mathrm{T} 4$ endonuclease $\mathrm{V}$ in liposomes on skin cancer in Xeroderma pigmentosum: a randomized study. Lancet, 2001, 357, 926-929.

[12] Lods, L.M.; Dress, C.; Jhhonson, C.; Scholz, D.B.; Brooks, G.J. The future of enzymes in cosmetics. Int. J. Cosmet. Sci., 2000, 22, 85-94

[13] Svobova, A.; Psotova, J.; Walterova, D. Natural phenolics in the prevention of UV induced skin damage. A Review. Biomed. Papers, 2003, 147(2), 137-145.

[14] Gasparro, F.P. Sunscreens, skin photobiology and skin cancer: the need for UVA protection and evaluation of efficacy. Environ. Health Perspect., 2000, 108(1), 71-78.

[15] Seite, S.; Bredoux, C.; Compan, D.; Zucchi, H.; Lombard, D; Medaisko, C; Fourtainer, A. Histological evaluation of atopically applied retinol- vitamin C combination. Skin Pharmacol. Physiol., 2005, 18, 81-87.

[16] Silva, M.R.; Carneiro, S.C.D.S. Elderly skin and its rejuvenation: products and procedures for the aging skin. J. Cosmet. Dermatol., 2007, 6, 40-50.

[17] Deep, C.; Saraf, S. Novel approaches in herbal cosmetics. J. Cosmet. Dermatol., 2008, 7, 89-95.

[18] Hoet P.H.M.; Irene, B.H.; Oleg, V.S. Nanoparticles- known and unknown health risks. J. Nanobiotechnol., 2006, 2, 12-27.

[19] Chen, H.; Weiss, J.; Shahidi, F. Nanotechnology in nutraceuticals and functional foods. Inodtechnology, 2006, 30.06, 30-36. Avilable from: http://www.ift.org

[20] Carlotti, M.E.; Gallarate, M.; Rossatto, V. O/W microemulsion as a vehicle for sunscreens. J. Cosmet. Sci., 2003, 54(5), 451-462.

[21] Gallarate, M.; Carlotti, M.E.; Trotta, M.; Bovo, S. On the stability of ascorbic acid in emulsified systems for topical and cosmetic use. Int. J. Pharm., 1999, 188(2), 233-241.

[22] Khan, A.Y.; Talegaonkar, S.; Iqbal, Z.; Ahmed, F.J.; Khar, R.K. Multiple emulsions: an overview. Curr. Drug Deliv., 2006, 3(4), 429-443.

[23] Okochi, H.; Nakano, M. Preparation and evaluation of w/o/w type emulsions containing vancomycin. Adv. Drug Deliv. Rev., 2000, 45, 5-26.

[24] Raynal, S.; Grossiord, J.L.; Seiller, M.; Clausse, D. A topical w/o/w multiple emulsion containing several active substances: formulation, characterization and study of release. J. Cont. Rel., 1993, 26, 129-140.

[25] Niemiec, S.M.; Ramachandran, C.; Weiner, N. Influence of nonionic liposomal composition on topical delivery of peptide drugs into pilosebaceous units: an in vivo study using the hamster ear model. Pharm Res., 1995, 12(8), 1184-1188.
[26] Cevc, G. Lipid vesicles and other colloids as drug carriers on the skin. Adv. Drug Deliv. Rev., 2004, 56(5), 675-711.

[27] Bombardelli, E.; Cristoni, A.; Morazzoni, P. Phytosomes in functional cosmetics. Fitoterpia. $L X V$, 1994, 5, 387-389.

[28] Bombardelli, E.; Cum, S.B.; Loggia, R.; Della, N.; Del, P.; Tubaro, A.; Gariboldi, P. Complexes between phospholipids and vegetal derivatives of biological interest. Fitoterpia, 1989, 60, 1-9.

[29] Loggia, R.D.; Sosa, S.; Tubaro, A.; Morazzoni, P.; Bombardelli, E.; Griffin, A. Anti-Inflammatory activity of some Gingko biloba constituents and of their phospholipids- complexes. Fitoterapia, 1996, 3, 257-273.

[30] Yanyu, X.; Yunmei, S.; Zhipeng, C.; Qineng, P. The preparation of silybin- phospholipid complex and the study on its pharmacokinetics in rats. Int. J. Pharma., 2006, 307, 77-82.

[31] Franco, P.G.; Bombardelli, E. Complex compound of bioflavonoids with phospholipids, their preparation and use and pharmaceutical and cosmetic composition containing them. US Patent, EPTO no. EP0275005, 1988, 6-14.

[32] Gupta, A.; Ashawat, M.S.; Saraf, S.; Saraf, S. Phytosome: a novel approach towards functional cosmetics. J. Plant Sci., 2007, 2(6), 644- 649.

[33] Benson, H.A. Transferosomes for transdermal drug delivery. Expert Opin. Drug Deliv., 2006, 3(6), 727-737.

[34] Cevc, G. Drug delivery across the skin. Expert Opin. Investig. Drugs, 1997, 6(12), 1887-1937.

[35] Schmid, D.; Zulli, F. Role of beta endorphin in the skin. Int. J. Appl. Sci., 2005, 4, 131-134.

[36] Pople, P.V.; Singh, K.K. Development and evaluation of topical formulation containing solid lipid nanoparticles of vitamin A. AAPS Pharm. Sci. Tech., 2006, 7(4), Article 91, $\mathrm{E}_{1}-\mathrm{E}_{7}$.

[37] Wissing, S.A.; Muller, R.H. The influence of solid lipid nanoparticles on skin hydration and viscoelasticity in vivo study. Eur. J. Pharm., 2003, 56(1), 67-72.

[38] Souto, E.B.; Muller, R.H.; Gohla, S. A novel approach based on lipid nanoparticles (SLN) for topical delivery of alpha- lipoic acid. J. Microencapsul., 2005, 22(6), 581-592.

[39] Wissing, S.A.; Muller, R.H. Cosmetic applications of solid lipid nanoparticles. Int. J. Pharm., 2003, 254(1), 65-68.

[40] Zulli, F. Preparation and properties of Coenzyme Q10 nanoemulsions. Cosmet. Sci. Technol., 2006, 1, 1-7.

[41] Merisko, L.E.; Liversidge, G.G.; Cooper, E.R. Nanosizing: a formulation approach for poorly-water-soluble compounds. Eur. J. Pharm. Sci., 2003, 18(2), 113-120.

[42] Da, Y.; Bruce, A.; Seth, R.M. Cubic liquid-crystalline nanoparticles. J. Nanoparticle Res., 2002, 4(4), 297-311.

[43] Embil, K.; Nacht, S. The microsponge delivery system (MDS): a topical delivery system with reduced irritancy incorporating multiple triggering mechanisms for the release of actives. J. Microencapsul., 1996, 13(5), 575-588.

[44] Chadawar, V.; Shaji, J. Microsponge delivery system. Curr. Drug Deliv., 2007, 4(2), 123-129.

[45] Felton, L.A.; Wiley, C.J.; Godwin, D. Influence of cyclodextrin complexation on the in vivo photoprotective effects of oxybenzone. Drug Dev. Indust. Pharm., 2004, 30, 95-102.

[46] Pissawini, M.; Ferrero, L. In vitro determination of Sun protection Factor. Bus. Brief. Glob. Cosmet. Manuf., 2004, 2, 1-5.

[47] Knott, A.; Koop, U.; Mielke, H.; Reuschlein, K.; Peters, N.; Muhr, G.M.; Lenz, H.; Wensorra, U.; Jaspers, S.; Kolbe, L.; Raschke, T.; Stäb, F.; Wenck, H.; Gallinat, S. A novel treatment option for photoaged skin. J. Cosmetic Dermatol., 2008, 7, 15-22.

[48] Ashawat, M.S.; Saraf, S.; Saraf, S. Preparation and characterization of herbal creams for improvement of skin viscoelastic properties. Int. J. Cosmet. Sci., 2008, 30(3), 183-193.

(C) Chanchal and Swarnlata; Licensee Bentham Open.

This is an open access article licensed under the terms of the Creative Commons Attribution Non-Commercial License (http://creativecommons.org/licenses/by-nc/3.0/) which permits unrestricted, non-commercial use, distribution and reproduction in any medium, provided the work is properly cited. 